\title{
Adult Diagnosis of Swyer-James-Macleod Syndrome: Retrospective Analysis of Four Cases
}

\author{
Hadice Selimoglu Sen MD, Mahsuk Taylan MD, Ozlem Abakay MD, \\ Cengizhan Sezgi MD, and Mehmet Guli Cetincakmak MD
}

\begin{abstract}
Swyer-James-Macleod syndrome (SJMS) is a rare constrictive bronchiolitis with air-flow obstruction and a decreased number and diameter of ipsilateral peripheral pulmonary vessels. This syndrome is characterized by unilateral hyperlucency on chest radiography. Computed tomography provides useful additional information. The diagnosis is usually made in childhood but sometimes occurs in adulthood. The disease often presents with dyspnea, decreased exercise tolerance, cough, hemoptysis, and recurrent pulmonary infections. SJMS may be confused with asthma or pulmonary embolism due to similar symptoms and may result in inappropriate therapy. This case series examined the clinical and imaging spectrum of four patients who were diagnosed with SJMS in adulthood. Key words: Swyer-James; Macleod; pulmonary artery hypoplasia. [Respir Care 2014;59(4):e51-e54. (C) 2014 Daedalus Enterprises]
\end{abstract}

\section{Introduction}

In 1953, Swyer and James and, in 1954, Macleod, who provided much more detail, described patients with unilateral hyperlucent lungs. Swyer-James-Macleod syndrome (SJMS), or unilateral hyperlucent lung syndrome, is a rare entity associated with post-infectious bronchiolitis obliterans occurring in childhood. ${ }^{1}$ This syndrome is a long-term complication of bronchiolitis in children, especially after adenoviral infection occurring in infancy. ${ }^{2}$ The affected child may be asymptomatic, but more often, the patient has recurrent pulmonary infections and develops bronchiectasis. Dyspnea, hemoptysis, and chronic productive cough are visible symptoms. A basic pathologic condition is bronchiolitis associated with obliteration of the small airways and a severe emphysematous pattern owing to

Drs Selimoglu Sen, Taylan, Abakay, and Sezgi are affiliated with the Department of Pulmonology, and Dr Cetincakmak is affiliated with the Department of Radiodiagnostics, Faculty of Medicine, Dicle University, Diyarbakir, Turkey.

The authors have disclosed no conflicts of interest.

Correspondence: Hadice Selimoglu Sen MD, Department of Pulmonology, Faculty of Medicine, Dicle University, 21280 Diyarbakir, Turkey. E-mail: dr.haticesen@hotmail.com.

DOI: $10.4187 /$ respcare. 02552 related alveolar destruction and dilated lung parenchyma. ${ }^{3}$ Peripheral pulmonary vascularization is decreased as a result of inflammation. Unilateral or bilateral involvement is possible. A chest radiograph demonstrates lobar or unilateral hyperlucent lung and normal or reduced volume of the affected lung. Air-flow obstruction is generally present in pulmonary function testing (PFT), and ventilation and perfusion scanning often reveals markedly decreased perfusion of the affected lung. ${ }^{4}$ The bronchographic findings are striking and limited to the abnormal lung. ${ }^{5}$ The major bronchi are normal, but the smaller branches are club-like, and occasionally small buds project from the ends of the peripheral divisions. Generally, there is almost a complete absence of alveolar filling, demonstrated by a well-demarcated clear zone between the smaller bronchiectatic bronchi and the chest wall. ${ }^{5}$ This disorder is typically diagnosed in childhood after an evaluation for recurrent respiratory infections, but patients who have little or no sequelae of bronchiectasis sometimes have minor symptoms or are asymptomatic and may therefore not be diagnosed until adulthood. ${ }^{6}$ The clinical and imaging spectrum of 4 patients who were diagnosed with SJMS in adulthood at a university hospital is presented in this case series. These patients were remarkable, with involvement of the left side accompanied by bronchiectasis. It is interesting that 3 patients were asymptomatic until adulthood even though they had bronchiectasis and obstructive defects in PFT. 

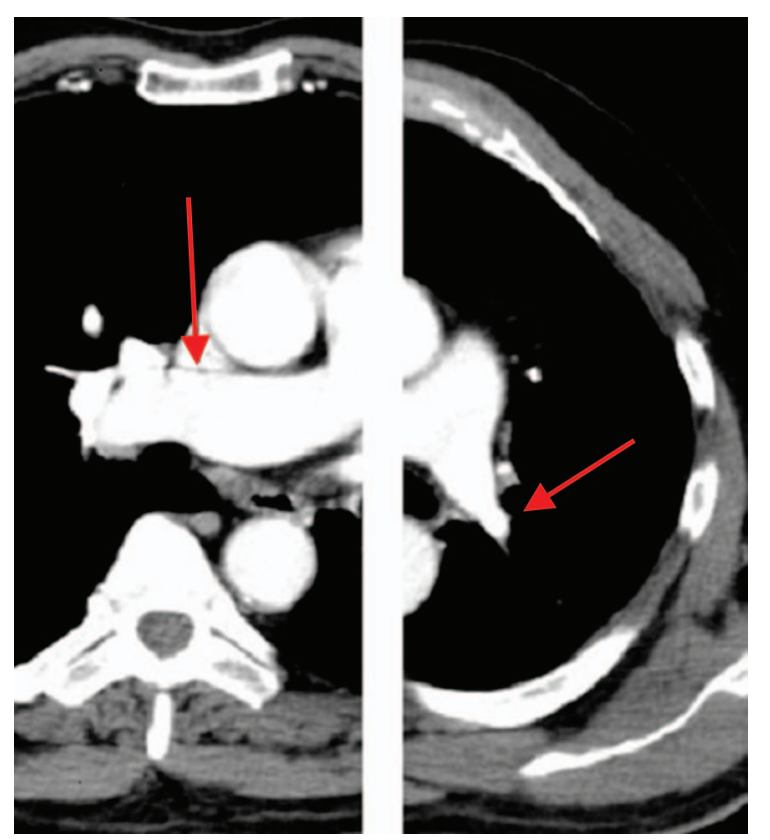

Fig. 1. Left: CT of Case 1 showing right main pulmonary artery at normal diameter (arrow). Right: CT of Case 1 showing left pulmonary artery hypoplasia (arrow).

\section{Case Report 1}

A 39-year-old male patient was admitted with complaints of dyspnea, cough, and hemoptysis. He had been admitted to different hospitals with complaints of cough and sputum production for $\sim 3$ years. Hemoptysis produced the amount of a teaspoon and was very rare. He had been treated for acute bronchitis with antibiotics and bronchodilators in the previous 3 years. He had no smoking history. There were no significant problems in childhood. Pulmonary auscultation demonstrated decreased breath sounds at the base of the left hemithorax. The chest radiograph on admission disclosed unilateral hyperlucency, bronchiectasis, and reduced volume in the left lung. Computed tomography (CT) scans demonstrated pulmonary artery hypoplasia, bronchiectasis, decrease in density, and volume loss in the left lung (Fig. 1). There was moderate obstruction as determined by PFT.

\section{Case Report 2}

A 51-year-old female patient was admitted with complaints of dyspnea, cough, chest pain, and hemoptysis. Her symptoms had started after a severe infection of the respiratory tract at the age of 11 years. The patient's symptoms decreased and disappeared from time to time but continued for $\sim 40$ years. There was moderate obstruction as determined by PFT with a diagnosis of COPD, and she was treated with a combination of inhaled long-acting

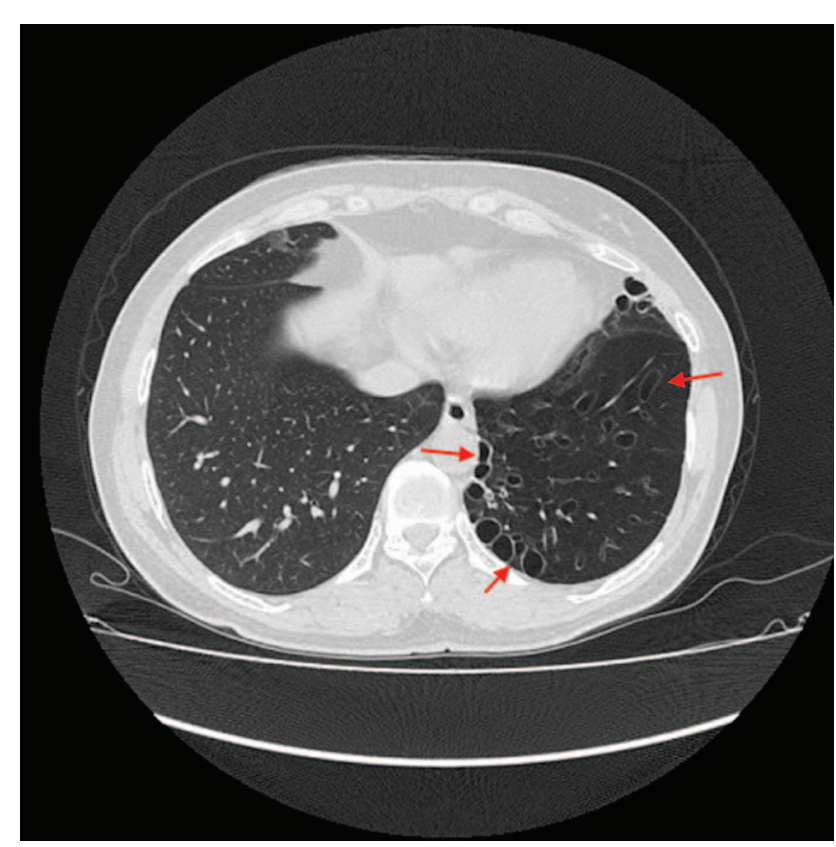

Fig. 2. CT of Case 2 showing widespread bronchiectasis in the left lung (arrows).

$\beta$-agonist and corticosteroid for a long time. She had no history of smoking. There were no significant problems in childhood. Subcrepitan crackles at the base of the left hemithorax with bilateral expiratory wheezes was noted on pulmonary auscultation. The chest radiograph on admission disclosed unilateral hyperlucency, bronchiectasis, and reduced volume in the left lung. CT scans demonstrated pulmonary artery hypoplasia, widespread bronchiectasis, decrease in density, and volume loss in the left lung (Fig. 2).

\section{Case Report 3}

A 40-year-old female was referred to us with complaints of cough, sputum, and chest pain that had continued for 3 years. Her medical history noted recurrent pulmonary infections for $\sim 3$ years and an unstudied chronic productive cough. The patient was positive for bilateral expiratory wheezes on physical examination. Chest radiographs showed hyperlucency of the left lung, with air trapping during expiration. CT scans demonstrated pulmonary artery hypoplasia, bronchiectasis, decrease in density, and volume loss in the left lung and a mosaic perfusion defect in the right lung (Fig. 3). PFT revealed mild airway obstruction with no response to bronchodilators.

\section{Case Report 4}

A 33-year-old female patient was referred to our hospital with a pre-diagnosis of difficult asthma. She was 


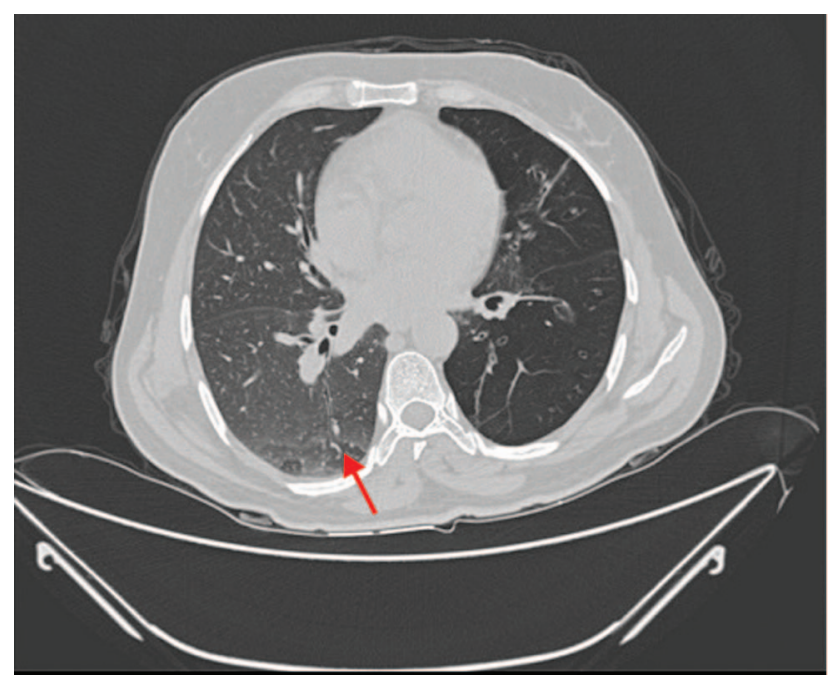

Fig. 3. CT of Case 3 showing mosaic perfusion in the left lung (arrow).

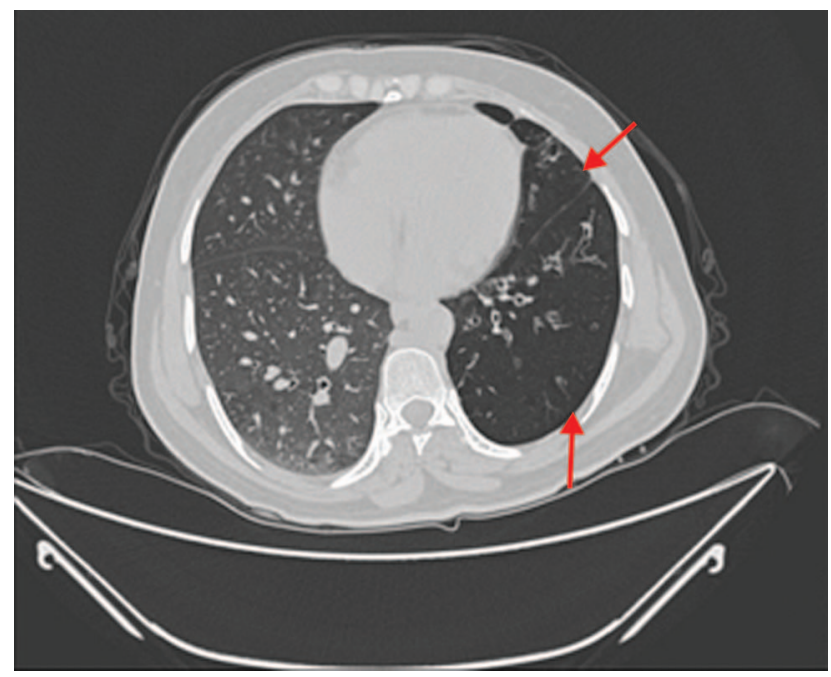

Fig. 4. CT of Case 4 showing low density areas in the left lung (arrows).

treated with bronchodilator and corticosteroid therapy for $\sim 6$ months but did not benefit from the treatment. Although her prominent symptom was dyspnea, she also has a small cough, sputum production, and chest pain. The patient was positive for bilateral expiratory wheezes and crepitant rales at the base of the left hemithorax on pulmonary auscultation. Chest radiographs showed hyperlucency of the left lung. CT scans demonstrated pulmonary artery hypoplasia, bronchiectasis, decrease in density, and volume loss on the left side and a mosaic perfusion defect on both the left and right sides (Fig. 4). PFT revealed moderate obstruction with response to bronchodilators and mild restriction.

\section{Discussion}

Unilateral bronchiolitis obliterans with hyperinflation is quite rare, with a prevalence of $0.01 \%$ in 17,450 surveyed chest radiographs. ${ }^{7}$ It is presently considered to be an acquired disease secondary to viral bronchiolitis and pneumonitis in childhood etiology associated with Paramyxovirus morbillivirus, Bordetella pertussis, Mycobacterium tuberculosis, Mycoplasma pneumoniae, influenza A, and adenovirus (types 3, 7, and 21). ${ }^{1}$ Interestingly, 3 of our patients did not have any history of significant respiratory infections in childhood. Clinically, the disease often presents with dyspnea, decreased exercise tolerance, cough, hemoptysis, and recurrent pulmonary infections. ${ }^{2}$ Dyspnea on exertion was the most frequent symptom in a series with 8 patients in contrast to an earlier study of 9 patients in which dyspnea was not a prominent feature. ${ }^{8,9}$ Our cases had one or more of these symptoms. Dyspnea and cough were the most frequent symptoms. Physical examination findings are nonspecific and may include decreased chest expansion, rales, or hyper-resonance. Abba and Al-Mobeireek $^{9}$ found that most adults with SJMS are symptomatic, often for a prolonged period of time at presentation. Three of our patients were asymptomatic for many years, although one had ongoing symptoms for nearly 40 years at intervals. Complications of unilateral hyperlucent lung syndrome include recurrent infection in areas of bronchiectasis, lung abscesses, and spontaneous pneumothorax. ${ }^{10,11}$

SJMS is characterized by unilateral hyperlucency in radiographs and air trapping. Chest radiography shows a normal or small lung with increased translucency owing to small hilar vessels that arborize sparsely and are attenuated. This typical chest radiography view was present in our cases, and it was a stimulus for further examination. Thorax CT and high-resolution CT provide useful additional information, such as patchy bilateral regions of hyperlucency or bronchiectasis. CT showed the typical findings of hyperlucent areas with diffuse oligemia and air trapping during expiration in two cases. These findings are known as a mosaic perfusion. Bronchiectasis is not necessary for diagnosis but is present in some cases, and there are several explanations for this. First, bronchiolitis obliterans can cause atelectasis or scarring, which in turn leads to bronchial dilatation. There was left-side bronchiectasis in our 4 cases. PFT showed obstructive defects in varied degrees. ${ }^{12}$ In this present study, there was obstruction in 4 patients and restriction in 1 patient on spirometric examination. Hypoxemia was not present in any patient as determined via pulse oximeter. Treatment of SJMS is typically conservative and supportive, including close follow-up and management of recurrent pulmonary infections and rarely including lung resections for recalcitrant disease. ${ }^{13}$ Only symptomatic treatment was needed for our patients. SJMS can be confused with many chronic lung 


\section{Adult Diagnosis of Swyer-James-Macleod Syndrome}

diseases due to similar symptoms and may result in inappropriate therapy. One of our patients had been treated for many years for COPD. Another case was referred to us with a pre-diagnosis of difficult asthma.

\section{Conclusions}

The main reason for reporting this case series is the rarity of patients who have been diagnosed with SJMS in adulthood. Although respiratory tract infection is considered to be a very important factor, as many as 3 of our patients had no history of airway infection in childhood. Therefore, some other genetic or environmental factors may contribute to the development of this syndrome.

\section{REFERENCES}

1. Tortajada M, Gracia M, García E, Hernández R. [Diagnostic considerations in unilateral hyperlucency of the lung (Swyer-JamesMacLeod syndrome)]. Allergol Immunopathol (Madr) 2004;32(5): 265-270. Article in Spanish.

2. Talmadge EK, Kinder BW. Bronchiolitis. In: Fishman AP, Elias JA, Fishman JA, Grippi MA, Senior RM, Pack AI, editors. Fishman's pulmonary diseases and disorders, volumes 1 and 2. Philadelphia: McGraw-Hill Medical; 2008:1423-1447.

3. Yekeler E. A rare case of Swyer-James Macleod syndrome and a new clinical presentation, acquired lobar emphysema. Ann Thorac Surg 2012;93(5):e123-e125.
4. Arslan N, Ilgan S, Ozkan M, Yuksekol I, Bulakbasi N, Pabuscu Y, et al. Utility of ventilation and perfusion scan in the diagnosis of young military recruits with an incidental finding of hyperlucent lung. Nucl Med Commun 2001;22(5):525-530.

5. Gottlieb LS, Turner AF. Swyer-James (Macleod's) syndrome. Variations in pulmonary-bronchial arterial blood flow. Chest 1976;69(1): 62-66.

6. Sulaiman A, Cavaille A, Vaunois B, Tiffet O. Swyer-JamesMacLeod syndrome; repeated chest drainages in a patient misdiagnosed with pneumothorax. Interact Cardiovasc Thorac Surg 2009; 8(4):482-484

7. Gaensler EA. Unilateral hyperlucent lung. In: Simon M, Potchen EJ, LeMay M, editors. Frontiers of pulmonary radiology. Orlando: Grune \& Stratton; 1969:312-359.

8. Marti-Bonmati L, Ruiz Perales F, Catala F, Mata JM, Calonge E. CT findings in Swyer-James syndrome. Radiology 1989;172(2):477-480.

9. Abba AA, Al-Mobeireek AF. Clinical spectrum of Swyer-JamesMacleod syndrome in adults. Saudi Med J 2003;24(2):195-198.

10. Wang JY, Chen KY, Chang YC, Chang YL, Lee LN. Swyer-James syndrome complicated by lung abscess. J Formos Med Assoc 2000; 99(3):252-256.

11. Soni R, Barnes D. Macleod's syndrome presenting with spontaneous pneumothorax. Respirology 1999;4(3):275-277.

12. Quaedvlieg V, Duysinx B, Ghaye B, Bartsch P. [Clinical case of the month. MacLeod syndrome]. Rev Med Liege 2004;59(12):691-694. Article in French.

13. Tutar O, Tekcan DE, Samanci C, Bas A. Adult diagnosis of SwyerJames-Macleod syndrome. BMJ Case Rep 2012. doi:10.1136/bcr2012-007349. 